\title{
线粒体沉默信息调节因子家族在癫痛发生发展中 的作用研究进展
}

朱 锋 ${ }^{1}$, 项迎春 ${ }^{2}$, 曾玲晖 ${ }^{1}$

1. 浙大城市学院医学院, 浙江杭州 310015

2. 浙江医院药剂科, 浙江杭州 310012

[摘 要] 线粒体沉默信息调节因子 (sirtuin)包括SIRT3、SIRT4和SIRT5, 在细胞 寿命、细胞调亡、基因组稳定性和代谢等进程中发挥重要的作用。SIRT3、SIRT4 和SIRT5 参与保护应激状态下线粒体的完整性, 维持其能量代谢, 调节脑内神经递 质受体、神经营养因子、细胞外基质蛋白及各种转录调节因子表达, 在遗传或获

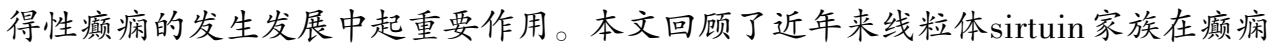
发病机制中的研究进展, 同时针对以线粒体 sirtuin为治疗靶点的相关问题进行总结 并展望,为进一步研究线粒体sirtuin功能和临床应用提供依据。

[关键词 ] 线粒体;沉默信息调节因子; 领痳;氧化应激;机制; 综述

[中图分类号 ］R742.1 [ 文献标志码 ］A

\section{Progress on mitochondrial silence information regulator family in epilepsy}

ZHU Feng ${ }^{1}$, XIANG Yingchun ${ }^{2}$, ZENG Linghui ${ }^{1}$ (1. School of Medicine, Zhejiang University City College, Hangzhou 310015, China; 2. Department of Pharmacy, Zhejiang Hospital, Hangzhou 310012 ,China)

Corresponding author: ZENG Linghui, E-mail: zenglh@zucc.edu.cn, https://orcid.org/ 0000-0002-5924-4419

\begin{abstract}
[ Abstract ] SIRT3, SIRT4 and SIRT5 are located in mitochondria and also known as mitochondrial sirtuins. They play important roles in regulating many cellular functions including cell survival, cell cycle or apoptosis, DNA repair and metabolism. Mitochondrial sirtuins are involved in the protection of mitochondrial integrity and energy metabolism under stress regulating the expression of neurotransmitter receptors, neurotrophins, extracellular matrix proteins and various transcription factors, thus involved in
\end{abstract}

\section{收稿日期: 2020-10-20 接受日期:2021-02-24 网络预发表日期:2021-06-15}

基金项目: 浙江省自然科学基金(LY20H09002); 浙江省医药卫生科技计划 (2020RC088); 浙江省实验动物科技计划 (2018C37131)

第一作者: 朱 锋,副教授,主要从事神经药理学研究;E-mail:zhuf@zucc.edu.cn;https://orcid.org/0000-0002-3220-6762 通信作者:曾玲晖,教授,博士生导师, 主要从事神经药理学研究;E-mail:zenglh@zucc.edu.cn; https://orcid.org/0000-00025924-4419 
epileptogenesis triggered by both genetic or acquired factors. Here we review research progress on the actions of mitochondrial sirtuin in epilepsy; and discuss the challenges and perspectives of mitochondrial sirtuin as a potential therapeutic target for epilepsy.

[ Key words ] Mitochondrion; Silence information regulator; Epilepsy; Oxidative stress; Mechanism; Review

[J Zhejiang Univ Med Sci, 2021, 50(3): 403-408.]

[缩略语] 沉默信息调节因子 (silence information regulator, sirtuin, SIRT); 烟酰胺腺 嘌呤二核苷酸 (nicotinamide adenine dinucleotide, NAD); 腺苷二磷酸 (adensine diphosphate, ADP); 超氧化物歧化酶 (superoxide dismutase, SOD); 异柠檬酸脱氢酶(isocitrate dehydrogenase, IDH); 过氧化物酶体增殖物激活受体 $\gamma$ 辅助激活因子 (peroxisome proliferators-activated receptors- $\gamma$ coactlvator, PGC); 解偶联蛋白 (uncoupling protein, UCP); 腺苷三磷酸 (adenosine triphosphate, ATP); 氨甲酰磷酸合成酶(carbamyl phosphate synthetase, CPS)

癫㾁是由神经元突发性异常放电导致的短暂 性大脑功能障碍疾病, 是一种常见且严重的神经 性疾病。癫㾁发作具有突然性、短暂性和反复性 等特点, 严重影响患者的生活质量 ${ }^{[1]}$ 。目前, 癫㾁 的发生机制尚未明确。越来越多的研究表明, 氧 化应激在癫㾁的发生发展中起到关键作用, 尤其 是线粒体功能障碍, 可能是难治性癫㾁的主要发 病机制 ${ }^{[2-4]}$ 。大脑因线粒体丰富, 需氧量高, 修复 能力差, 极易受到氧化损伤。癫㾁发生过程中, 大 脑神经元异常放电, 造成急性脑损伤, 诱发活性氧 堆积, 同时线粒体呼吸链活性下降 ${ }^{[5]}$ 。而线粒体 功能异常及大脑中活性氧无法及时清除可引起氧 化应激, 进一步加重癫㾁的发生 ${ }^{[6]}$ 。研究发现, 抗 氧化剂可以缓解动物癫㾁模型的认知缺陷, 降低 持续癫㾁状态引起的病死率 ${ }^{[7]}$ 。线粒体是产生活 性氧的主要部位, 是氧化应激的主要靶标, 深人研 究癫㾁发生发展过程中线粒体的抗氧化防御机制 具有重要意义。

sirtuin 家族是一组高度保守的 NAD 依赖蛋白 质去乙酰化酶, 其主要功能是对蛋白质翻译后进 行乙酰化修饰,还包括ADP核糖基化、去丙二酰化 和酰胺化等修饰, 在细胞寿命、细胞调亡、基因组 稳定性和代谢等进程中发挥广泛而重要的作 用 ${ }^{[8]}$ 。目前, 在人类中已经鉴定出 7 个 sirtuin 家族 同源类似物, 分别命名为 SIRT1 SIRT7, 其在组织 分布、酶活性、亚细胞定位、生化功能和靶蛋白上 均具有一定差异。其中SIRT1、SIRT6和SIRT7 主
要位于细胞核,SIRT2 主要位于细胞质,SIRT3、 SIRT4、SIRT5 主要位于线粒体 ${ }^{[9]}$ 。线粒体作为细 胞的 “能量工厂” 具有很多重要的生理功能, 其 功能障碍与癫㾁神经元选择易损性密切相关, 可 能是癫㾁发病的核心机制 ${ }^{[10]}$ 。近年来主要定位 在线粒体的SIRT3、SIRT4 和SIRT5 在癫㾁研究领 域备受关注。本文就线粒体sirtuin在癫㾁发生发 展中的最新研究进展进行综述。

\section{SIRT3 通过去乙酰化降低线粒体氧化还原水 平调控癫㾋的发生发展}

SIRT3基因位于人类第 11 号染色体(11p15.5), 由 21902 个碱基组成。多数研究显示 SIRT3 位于 线粒体内膜脊和基质上, 但也有部分研究认为其 位于细胞核和细胞质中 ${ }^{[11]}$ 。sirtuin 家族中 SIRT3 去乙酰化酶活性最强, 其分布与组织代谢的强度 具有较强的关联性, 在代谢旺盛的脑、肝脏及心脏 中大量表达, 可广泛调控线粒体的形态和功能, 在 能量代谢、氧化应激等方面发挥重要作用 ${ }^{[12]}$ 。线 粒体蛋白质组学分析确定了约 100 种可能受 SIRT3调节的蛋白质,这些SIRT3 底物包括线粒体 电子传递链组分、线粒体 SOD2、线粒体通透性转 变通道诱导蛋白亲环素 $\mathrm{D}$ 、脂肪酸 $\beta$ 氧化、氨基酸 代谢等 ${ }^{[13-15]}$ 。

SIRT3 通过去乙酰化线粒体蛋白降低线粒体 氧化还原水平, 稳定细胞和线粒体的钙稳态, 可保 护神经元, 抑制调亡。研究发现, 幼年癫㾁大鼠海 
马中SIRT3 表达降低, 而干预SIRT3 表达后, 其海 马线粒体肿胀、嵴变形 ${ }^{[16]}$ 。基于质谱的海马线粒 体乙酰化组学研究结果提示, SIRT3 功能障碍和异 常蛋白乙酰化可能是慢性癫㾁患者线粒体功能障 碍的原因之一 ${ }^{[17]}$ 。SOD2 是线粒体的主要抗氧化 酶,SIRT3 可以直接去乙酰化并激活抗氧化因子锰 SOD 和 IDH 2 , 提高线粒体对活性氧的清除能力并 降低活性氧水平。Cho 等 ${ }^{[18]}$ 研究结果表明, 与野 生型小鼠相比,SIRT3敲除小鼠中由红藻氨酸引发 的海马神经元死亡现象更加显著。海马神经元线 粒体内SIRT3 在癫㾁发作急性期一过性增高, 发挥 作用后转移至细胞核内。SIRT3 通过直接激活 SOD2保护海马细胞免受活性氧的损伤, 提示SIRT3 是一个潜在的有效治疗靶点, 可延缓自发性癫㾁的 发生。Gano 等 ${ }^{[17]}$ 通过红藻氨酸诱导的大鼠颞叶癫 㾁模型也发现海马区 SIRT3 表达水平先升后降的 趋势, 前期可能为应对癫㾁损伤的代偿性增加以消 除自由基,后期为损伤后 SIRT3 减少致使 SOD2、 IDH2 和线粒体电子传递链复合物 I 乙酰化水平增 高, 导致细胞内活性氧清除和能量供应功能障碍。

皮层神经元缺乏SIRT3 也易受到谷氨酸介导 的钙超载、兴奋毒性和氧化应激损伤 ${ }^{[19]}$ 。最新研 究表明,SIRT3 表达下降使大脑皮层中 $\gamma$ 氨基丁酸 能中间神经元易退化, 进而增强小清蛋白中间 神经元对红藻氨酸诱导急性 DNA 损伤的敏感 性 ${ }^{[20]}$ 。亲环素 D 在触发线粒体介导的调亡中起关 键作用, 可能介导了SIRT3对小清蛋白和钙视网膜 蛋白中间神经元的保护作用。SIRT3 可通过去乙 酰化亲环素 $\mathrm{D}$ 关闭线粒体通透性转换孔, 维持线粒 体的稳定性。SIRT3也可通过去乙酰化, 阻止促调 亡蛋白 Bax 由细胞核转运至线粒体, 从而抑制细胞 调亡 ${ }^{[21]}$ 。SIRT3 也可通过去乙酰化转录因子叉头 框蛋白 $O$ 提升 DNA 修复和抗氧化应激的能力, 减 弱其诱导的细胞调亡 ${ }^{[22]}$ 。

此外,SIRT3 还能够增强PGC1 $\alpha 、 U C P 1$ 等相关 线粒体蛋白基因的表达,调控线粒体活性氧生成以 及线粒体生物合成 ${ }^{[23]}$ 。Hasan-Olive 等 ${ }^{[24]}$ 在进行 生酮饮食抑制癫㾁发作机制研究中, 诱导线粒体 DNA 修复酶尿嘧啶-DNA 糖苷酶 1 表达突变, 选择 性地诱发小鼠前脑神经元线粒体功能障碍, 发现动 物生酮饮食或细胞暴露于酮体中可通过 PGC1 $\alpha$ SIRT3-UCP2 轴引发代偿机制, 改善线粒体的质量 及其生物能量, 有利于减少难治性癫痛发作。
由此可见,SIRT3 可以通过去乙酰化降低线 粒体氧化还原水平等机制调控癫㾋的发生发展, 有望为癲痫治疗带来新的希望。

\section{SIRT4 通过调节谷氨酸代谢在癫㾁中发挥神 经保护作用}

SIRT4 主要定位于线粒体,也具有典型的 NAD 依赖性去乙酰化酶活性。同时,SIRT4在谷氨 酰胺代谢和脂肪酸代谢等生物学过程中表现出 ADP 核糖基转移酶、脱酰基酶和脂酰胺酶等活性, 在细胞应激反应、能量稳态、代谢、基因组稳定性 及肿瘤的发生发展中具有重要作用 ${ }^{[25]}$ 。但SIRT4 的催化活性较弱,且具有明显的底物特异性。

SIRT4 可以调节新陈代谢和能量状态, 而大 脑中的许多过程如防止神经元细胞损伤和神经细 胞间信息传递等均依赖于 ATP,因此推测 SIRT4在 大脑功能发挥过程中具有关键的作用。SIRT4能 将 $\mathrm{NAD}$ 的 $\mathrm{ADP}$ 核糖基转移到谷氨酸脱氢酶残基 上,最终抑制细胞线粒体内的谷氨酰胺代谢, 降低 线粒体的氧化能力,减少 ATP 的产生 ${ }^{[26]}$ 。此外,谷 氨酸难以通过血脑屏障, 大脑严重依赖神经元及 神经胶质细胞膜上的谷氨酸转运体来清除突触上 多余的谷氨酸盐。而SIRT4能抑制谷氨酰胺和谷 氨酸的代谢,影响由谷氨酸累积引起兴奋性毒性 的神经系统疾病病理过程 ${ }^{[27]}$ 。Shih 等 ${ }^{[28]}$ 报道, SIRT4基因敲除小鼠在红藻氨酸诱导癫㾁发作中 症状加重, SIRT4的缺失导致谷氨酸摄取减少, 细 胞表面谷氨酸转运体表达减少; 而野生型小鼠在 抗癫㾁治疗 $24 \mathrm{~h}$ 后海马区SIRT4 水平升高。这些 结果提示, SIRT4 可促进谷氨酸摄取, 缓解神经兴 奋性毒性, 这种神经保护作用对癫㾁防治具有重 要的临床价值。

\section{SIRT5 通过调控神经系统内氨的水平及嘌呤 信号的转导影响癫㾋的发生发展}

SIRT5 蛋白主要定位于线粒体,由 14 个 $\alpha$ 环和 9 条 $\beta$ 链构成锌离子结构域和 Rossman 折叠域, 形 成底物和 NAD结合位点。SIRT5 具有与赖氨酸酰 基结合的结构特点,除具有较弱的去乙酰化酶活 性外, 还具有很强的去琥珀酰化、去丙二酰化和去 戊二酰基化酶活性,参与调控葡萄糖、氨基酸、脂 质等多种物质代谢过程, 并发挥抗氧化、调控细胞 和线粒体调亡、调节炎性反应等生物学功能 ${ }^{[29]}$ 。 
SIRT5 在各组织中分布广泛, 其在脑组织中 含量较高 ${ }^{[30]}$ 。SIRT5 在抑制线粒体活性氧水平中 具有重要作用, 是应激状态下神经元存活的 积极调控者 ${ }^{[31]}$, 参与多种神经系统疾病的发生 发展 ${ }^{[32]}$ 。

CPS1 是尿素循环的限速酶, 而尿酸氧化酶将 尿酸转换为尿囊素, 最终转化为尿素排出。SIRT5 可脱去 CPS 1 和尿酸氧化酶的乙酰基并上调其活 性, 促进尿素循环, 参与氨的解毒 ${ }^{[33]}$ 。尿素循环 主要发生在肝脏, 但Polletta等 ${ }^{[34]}$ 观察到SIRT5 在 非肝细胞中可通过调控谷氨酰胺代谢来调节氨的 产生和氨诱导的自噬。SIRT5 通过下调谷氨酰胺 酶的琥珀酰化水平抑制其活性, 进而减少谷氨酰 胺存在时氨的释放。在与氨积累有关的病理情况 下,SIRT5 激活可能有助于减少对氨积累敏感的细 胞 (如星形胶质细胞)的损害。SIRT5 在神经元中 的定位表明,谷氨酰胺酶可能是SIRT5 的蛋白质靶 点, 而不仅仅局限于星形胶质细胞的谷氨酰胺合 成酶 ${ }^{[35]}$ 。氨介导的独立于哺乳动物雷帕霉素靶 蛋白和 UNC-5 1 样激酶 $1 / 2$ 的自噬可能具有神经 保护作用 ${ }^{[36]}$ 。谷氨酸-谷氨酰胺循环与癫痫的关 系一直是人们关注的焦点 ${ }^{[37]}$ 。这些研究均暗示 了SIRT5 与癫㾋之间具有密切关系。

有研究将SIRT5 缺失小鼠和野生型小鼠暴露 于红藻氨酸中, 结果发现 SIRT5 缺失显著加重癫㾁 发作严重程度, 增加小鼠病死率, 加剧海马神经元 的死亡和退化, 并引发更加严重的反应性星形胶 质细胞增生 ${ }^{[38]}$ 。而在残存的海马线粒体中观察到 SIRT5 的表达相对上调, 这表明SIRT5 对细胞存活 率的提高有潜在贡献, 可能具有神经保护作用。 但该研究中SIRT5 缺失似乎并没有明显改变红藻 氨酸暴露引起的海马抗氧化酶 SOD2 和谷胱甘肽 过氧化物酶的下降, 其对癫痫发作和细胞退行性 变的保护作用可能是通过其他调节机制实现的。

另一个关键发现是SIRT5 在嘌呤代谢调节中 的潜在作用 ${ }^{[35]}$ 。嘌呤代谢物是三磷酸脱氧核糖 核苷酸及三磷酸核糖核苷酸的前体, 是DNA 合成 和修复、能量传输、神经传递和大脑中其他信号机 制的基本分子,其从头合成来源于葡萄糖衍生的丝 氨酸和磷酸戊糖途径产生的 5-磷酸核糖。SIRT5 介导的去戊二酰基化酶作用能增加葡萄糖 6 磷酸 脱氢酶 (磷酸成糖途径中的限速酶) 的活性, 有助 于提高大脑的抗氧化能力。近期研究显示, 星形
胶质细胞嘌呤信号转导的异常促进了癫㾁中神经 元的异常活动 ${ }^{[39]}$ 。

\section{4 展 望}

综上所述, 线粒体 sirtuin 家族参与了癫㾁发 生发展的多个病理生理过程,其在不同病理生理 条件下可能调控不同的下游相关信号通路, 确切 的作用机制及相互间的调节作用尚不明确。鉴于 不同线粒体 sirtuin蛋白在底物或修饰位置上的特 异性和重叠性, 其在不同代谢途径中对蛋白质功 能或酶活性提供协同或相反的作用 ${ }^{[40]}$ 。深人研 究线粒体sirtuin对癫㾁的治疗和预防具有深远意 义,特别是要考虑到不同线粒体sirtuin之间的相互 关联作用,其各自独立的、相互间协同或代偿的作 用均会影响疾病的发生发展。

笔者团队通过红藻氨酸诱导的小鼠癫㾁模型 发现,SIRT3基因敲除小鼠癫㾁发作潜伏时间明显 增加,发作严重程度明显下降,发作时间明显缩短 (待发表), 提示SIRT3 基因敲除对癫㾁有保护作 用。该结果与之前的报道不一致 ${ }^{[18]}$ 。为了进一 步确认其机制, 研究人员在癫㾁发作 $24 \mathrm{~h}$ 后检测 各组小鼠海马组织内SIRT3、SIRT4 和SIRT5 信使 RNA 表达情况, 结果显示, 在红藻氨酸致㾁后, SIRT3 在野生型小鼠中的表达明显减少,SIRT5 在 SIRT3基因敲除小鼠和野生型小鼠中的表达均减 少,SIRT4在野生型小鼠中的表达减少, 而在SIRT3 基因敲除小鼠中的表达增加, 提示 SIRT3 与 SIRT4 之间存在着一定相互作用。有学者发现,在心肌 缺血再灌注模型中敲除SIRT3后,并未发现心脏线 粒体中活性氧生成增多和蛋白质氧化增加, 而发 现SIRT4 表达上调 ${ }^{[41]}$,笔者团队研究结果与其一 致。这些研究进一步提示在不同的疾病模型中, 单纯以 SIRT3 作为药物研发的靶点可能存在局限 性, 其作用的发挥还可能与 SIRT4 密切关联。因 此,未来的研究须更深人、全面地了解各线粒体 sirtuin之间的相互关系, 为线粒体sirtuin作为治疗 靶点提供更多的科学依据, 从而为药物干预治疗 和预防癫㾁发作奠定基础。

利益冲突 所有作者均声明不存在利益冲突

\section{参考文献}

[1] KHAN A U, AKRAM M, DANIYAL M, et al. Awareness and current knowledge of epilepsy[J]. Metab Brain 
Dis, 2020, 35(1): 45-63.

[2] TERrone G, BALOSSO S, PAULETTI A, et al. Inflammation and reactive oxygen species as disease modifiers in epilepsy $[\mathrm{J}]$. Neuropharmacology, 2020, 167: 107742.

[3] LÖSCHER W, POTSCHKA H, SISODIYA S M, et al. Drug resistance in epilepsy: clinical impact, potential mechanisms, and new innovative treatment options $[\mathrm{J}]$. Pharmacol Rev, 2020, 72(3): 606-638.

[4] MANFORD M. Recent advances in epilepsy[J]. J Neurol, 2017, 264(8): 1811-1824.

[5] PEARSON-SMITH J N, PATEL M. Metabolic dysfunction and oxidative stress in epilepsy[J]. Int J Mol Sci, 2017, 18(11): 2365.

[6] SHEKH-AHMAD T, KOVAC S, ABRAMOV A Y, et al. Reactive oxygen species in status epilepticus[J]. Epilepsy Behav, 2019, 101: 106410.

[7] SHEKH-AHMAD T, LIEB A, KOVAC S, et al. Combination antioxidant therapy prevents epileptogenesis and modifies chronic epilepsy[J]. Redox Biol, 2019, 26: 101278.

[8] FRYDZISKA Z, OWCZAREK A, WINIARSKA K. Sirtuins and their role in metabolism regulation $[\mathrm{J}]$. Postepy Biochem, 2019, 65(1): 31-40.

[9] SINGH C K, CHHABRA G, NDIAYE M A, et al. The role of sirtuins in antioxidant and redox signaling $[\mathrm{J}]$. Antioxidants Redox Signal, 2018, 28(8): 643-661.

[10] ANAMIKA, KHANNA A, ACHARJEE P, et al. Mitochondrial SIRT3 and neurodegenerative brain disorders $[\mathrm{J}]$. J Chem Neuroanatomy, 2019, 95: 43-53.

[11] RAHMAN S. Mitochondrial diseases and status epilepticus $[\mathrm{J}]$. Epilepsia, 2018, 59: 70-77.

[12] WANG S, ZHANG J, DENG X, et al. Advances in characterization of SIRT3 deacetylation targets in mitochondrial function $[\mathrm{J}]$. Biochimie, 2020, 179: $1-13$.

[13] CARRICO C, MEYER J G, HE W, et al. The mitochondrial acylome emerges: proteomics, regulation by sirtuins, and metabolic and disease implications $[\mathrm{J}]$. Cell Metab, 2018, 27(3): 497-512.

[14] SOL E M, WAGNER S A, WEINERT B T, et al. Proteomic investigations of lysine acetylation identify diverse substrates of mitochondrial deacetylase Sirt3[J/OL]. PLoS ONE, 2012, 7(12): e50545.

[15] HALLOWS W C, YU W, SMITH B C, et al. Sirt3 promotes the urea cycle and fatty acid oxidation during dietary restriction $[\mathrm{J}]$. Mol Cell, 2011, 41(2): 139149.

[16] WU G, LIU J, LI S, et al. Glycyrrhizic acid protects juvenile epileptic rats against hippocampal damage through activation of Sirtuin3 $[\mathrm{J}]$. Brain Res Bull, 2020, 164: 98-106.
[17] GANO L B, LIANG L P, RYAN K, et al. Altered mitochondrial acetylation profiles in a kainic acid model of temporal lobe epilepsy[J]. Free Radical Biol Med, 2018, 123: 116-124.

[18 ] CHO I, JEONG K H, ZHU J, et al. Sirtuin3 protected against neuronal damage and cycled into nucleus in status epilepticus model[J]. Mol Neurobiol, 2019, 56(7): 4894-4903.

[19] CHENG A, YANG Y, ZHOU Y, et al. Mitochondrial SIRT3 mediates adaptive responses of neurons to exercise and metabolic and excitatory challenges $[\mathrm{J}]$. Cell Metab, 2016, 23(1): 128-142.

[20] CHENG A, WANG J, GHENA N, et al. SIRT3 haploinsufficiency aggravates loss of GABAergic interneurons and neuronal network hyperexcitability in an alzheimer's disease $\operatorname{model}[J]$. J Neurosci, 2020, 40(3): 694-709.

[21] DIKALOVA A E, ITANI H A, NAZAREWICZ R R, et al. Sirt3 impairment and SOD2 hyperacetylation in vascular oxidative stress and hypertension $[\mathrm{J}]$. Circ Res, 2017, 121(5): 564-574.

[22] SHUKLA S, SHARMA A, PANDEY V K, et al. Concurrent acetylation of FoxO1/3a and p53 due to sirtuins inhibition elicit Bim/PUMA mediated mitochondrial dysfunction and apoptosis in berberinetreated HepG2 cells[J]. Toxicol Appl Pharmacol, 2016, 291: 70-83.

[23 ] YIN J, NIELSEN M, CARCIONE T, et al. Apolipoprotein $\mathrm{E}$ regulates mitochondrial function through the PGC-1 $\alpha$-sirtuin 3 pathway[J]. Aging, 2019, 11(23): 11148-11156.

[24 ] HASAN-OLIVE M M, LAURITZEN K H, ALI M, et al. A ketogenic diet improves mitochondrial biogenesis and bioenergetics via the PGC1 $\alpha$-SIRT3-UCP2 axis $[J]$. Neurochem Res, 2019, 44(1): 22-37.

[25] HAN Y, ZHOU S, COETZEE S, et al. SIRT4 and its roles in energy and redox metabolism in health, disease and during exercise $[\mathrm{J}]$. Front Physiol, 2019, 10: 1006.

[26] LI Y, ZHOU Y, WANG F, et al. SIRT4 is the last puzzle of mitochondrial sirtuins $[\mathrm{J}]$. BioOrg Med Chem, 2018, 26(14): 3861-3865.

[27] SASAKI Y. Metabolic aspects of neuronal degeneration: From a $\mathrm{NAD}^{+}$point of view $[\mathrm{J}]$. Neurosci Res, 2019, 139: 9-20.

[28] SHIH J, LIU L, MASON A, et al. Loss of SIRT4 decreases GLT-1-dependent glutamate uptake and increases sensitivity to kainic $\operatorname{acid}[\mathrm{J}]$. J Neurochem, 2014, 131(5): 573-581.

[29] BRINGMAN-RODENBARGER L R, GUO A H, LYSSIOTIS C A, et al. Emerging roles for SIRT5 in metabolism and cancer $[\mathrm{J}]$. Antioxidants Redox Signal, 2018, 28(8): 677-690. 
[30] DU Y, HU H, HUA C, et al. Tissue distribution, subcellular localization, and enzymatic activity analysis of human SIRT5 isoforms $[\mathrm{J}]$. Biochem BioPhys Res Commun, 2018, 503(2): 763-769.

[31] KUMAR S, LOMBARD D B. Functions of the sirtuin deacylase SIRT5 in normal physiology and pathobiology $[\mathrm{J}]$. Crit Rev Biochem Mol Biol, 2018, 53(3): 311-334

[32] JESKO H, WENCEL P, STROSZNAJDER R P, et al. Sirtuins and their roles in brain aging and neurodegenerative disorders $[\mathrm{J}]$. Neurochem Res, 2017, 42(3): 876-890.

[33] NAKAGAWA T, LOMB D J, HAIGIS M C, et al. SIRT5 deacetylates carbamoyl phosphate synthetase 1 and regulates the urea cycle[J]. Cell, 2009, 137(3): 560-570.

[34] POLLETTA L, VERnUCGI E, CARNEVALE I, et al. SIRT5 regulation of ammonia-induced autophagy and mitophagy $[\mathrm{J}]$. Autophagy, 2015, 11(2): 253-270.

[35] KORONOWSKI K B, KHOURY N, MORRIS-BLAN$\mathrm{CO} \mathrm{K} \mathrm{C}$, et al. Metabolomics based identification of SIRT5 and protein kinase $\mathrm{C}$ epsilon regulated pathways in brain $[\mathrm{J}]$. Front Neurosci, 2018, 12: 32.

[36] LU K, ZIMMERMANN M, GÖRG B, et al. Hepatic encephalopathy is linked to alterations of autopha- gic flux in astrocytes [J]. EBioMedicine, 2019, 48: 539-553.

[37] EID T, GRUENBAUM S E, DHAHER R, et al. The glutamate-glutamine cycle in epilepsy[J]. Adv Neurobiol, 2016, 13: 351-400.

[38 ] LI F, LIU L. SIRT5 deficiency enhances susceptibility to kainate-induced seizures and exacerbates hippocampal neurodegeneration not through mitochondrial antioxidant enzyme SOD2[J]. Front Cell Neurosci, 2016, 10: 171

[39] NIKOLIC L, NOBILI P, SHEN W, et al. Role of astrocyte purinergic signaling in epilepsy[J]. Glia, 2020, 68(9): 1677-1691.

[40] WANG C H, WEI Y H. Roles of mitochondrial sirtuins in mitochondrial function, redox homeostasis, insulin resistance and type 2 diabetes $[\mathbf{J}]$. Int $\mathbf{J}$ Mol Sci, 2020, 21(15): 5266.

[41] PARODI-RULLÁN R M, CHAPA-DUBOCQ X, RULLÁN P J, et al. High sensitivity of SIRT3 deficient hearts to ischemia-reperfusion is associated with mitochondrial abnormalities $[\mathrm{J}]$. Front Pharmacol, 2017, 8: 275.

$$
\text { [本文编辑 李 璐 余 方] }
$$

\section{李君教授团队合作成果推出乙型肝炎肝衰竭预警预测系统2.0}

针对东西方人群乙型肝炎相关慢加急性肝衰竭 (HBV-ACLF) 患者的不同临床特征, 浙江大学传染病诊治国家重点 实验室李君教授团队前期已联合国内十三家医院肝病中心开展了一项多中心、前瞻性、开放性大队列研究,建立了适合 乙型肝炎人群的HBV-ACLF诊断的中国标准 (COSSH-ACLF) 和预后评分系统 (COSSH-ACLFs), 并揭示乙型肝炎恶化引起 的免疫代谢失衡是HBV-ACLF发生发展及重症化转归的关键机制, 相关研究结果已分别发表在《胃肠道》(Gut) 上。

研究人员在前期基础上进一步利用该前瞻性、多中心、COSSH开放研究大队列,发现年龄 (age)、肝性脑病 (HE)、总 胆红素 $(\mathrm{TB}) 、$ 、国际标准化比值 $(\mathrm{INR}) 、$ 血尿素 $(\mathrm{Ur})$ 和中性粒细胞计数 $(\mathrm{NC})$ 为HBV-ACLF患者 28 d预后的独立危险因素,据 此建立了HBV-ACLF新预后评分系统(COSSH-ACLF II s), 即 $1.649 \times \ln (\mathrm{INR})+0.457 \times \mathrm{HE}+0.425 \times \ln (\mathrm{NC})+0.396 \times \ln (\mathrm{TB})+$ $0.576 \times \ln (\mathrm{Ur})+0.033 \times \mathrm{age}$ 。相比先前的COSSH-ACLFs 和欧洲CLIF-ACLFs等常用的预后评分系统,基于临床指标实测值的 COSSH-ACLF II s 评分系统极大简化了临床计算及评估, 且具有更高的灵敏度和特异度, 以便临床医生及时床旁应用。研 究结果于2021年6月3日以 “Development and validation of a new prognostic score for hepatitis B virus-related acute-on-chronic liver failure” 为题在线发表在《肝病学杂志》(Journal of Hepatology)上(https://doi. org $/ 10.1016 / j$. jhep. 2021. 05. 026)。

李佳琪硕士、梁茜助理研究员、游绍莉主任医师、冯婷婷主治医师、周金硕士和朱冰副主任医师为共同第一作者,研 究工作得到国家自然科学基金、国家科技重大专项等资助。 